\title{
Plastic relaxation of metamorphic single layer and multilayer InGaAs/GaAs structures
}

\author{
D. J. Dunstan and P. Kidd \\ Strained-Layer Structures Research Group, Department of Physics, University of Surrey, Guildford, Surrey, \\ GU2 5XH, England \\ P. F. Fewster and N. L. Andrew \\ Philips Research Laboratory, Cross Oak Lane, Redhill, Surrey, England \\ R. Grey and J. P. R. David \\ SERC III-V Semiconductor Facility, Department of Electronic and Electrical Engineering, University \\ of Sheffield, P. O. Box 600, Mappin Street, Sheffield, S1 4DU, England \\ L. González and Y. González \\ Centro Nacional de Microelectronica. Serrano 144, 28006 Madrid, Spain
}

\section{A. Sacedón and F. González-Sanz \\ ETSI Telecomunicación, Dpto. Ingenería Electrónica, Universidad Politechnica de Madrid, 28040 Madrid, Spain}

(Received 8 January 1993; accepted for publication 1 June 1994)

\begin{abstract}
The plastic relaxation of multilayer structures of strained InGaAs grown above critical thickness on $\mathrm{GaAs}$ is reported and compared with the relaxation of single layers and with theory. We show that a composite structure, taken as a whole, follows the same relaxation law as observed in single layers. However, departures of the strains of some component layers from theory show that misfit dislocations are easily pinned at an interface. Implications for the design of relaxed buffer layer growth are discussed.
\end{abstract}

In semiconductor epitaxy, relaxed buffer layers are usually composed of several strained layers of different lattice constants. The analysis of the plastic relaxation of composite structures is neither theoretically nor experimentally well established. In this letter, we show how the behavior of composite structures can be predicted on the basis of an empirical single relaxation curve. ${ }^{1}$ We test the theory in simple multilayer structures. Previously, we found that the single curve was followed by $\operatorname{In}_{0.2} \mathrm{Ga}_{0.8} \mathrm{As} / \mathrm{GaAs}$ and $\mathrm{In}_{0.05} \mathrm{Ga}_{0.95} \mathrm{As} / \mathrm{GaAs} ;^{1}$ here we report new data for $\mathrm{In}_{0.1} \mathrm{Ga}_{0.9} \mathrm{As} / \mathrm{GaAs}$ and again find good agreement with the curve. The key result reported in this letter, however, is that multilayer structures as a whole also obey the theory. This is the first time that the plastic relaxation of composite epitaxial structures has been successfully predicted by theory. The residual strains of the individual layers in the structure deviate from the predicted values and this is interpreted in terms of dislocation pinning at the interface.

Relaxed buffer layers are needed for the growth of devices with lattice constants different from the available substrates; an example is the metamorphic InGaAs high electron mobility transistor (HEMT) reported by Win et al. ${ }^{2}$ Ideally, buffer layer structures should have no residual strain: onc of the major problems with GaAs on silicon technology is the presence of about 1 kbar residual stress in the GaAs which can destabilize the structure during subsequent growth or processing. ${ }^{3}$ In order to design fully strain-relieved structures, it is necessary to know the plastic relaxation which each constituent layer should undergo, and whether this is affected by other layers in the structure. We have therefore studied the simplest multilayer structures consisting of a first layer of $\mathrm{In}_{x 1} \mathrm{Ga}_{1-x 1}$ As of thickness $d_{1}$ and low mismatch strain $\epsilon_{01}=0.07 x_{1}$, followed by a second layer of
$\mathrm{In}_{x 2} \mathrm{Ga}_{1-x 2} \mathrm{As}$ of thickncss $d_{2}$ and higher mismatch strain $\epsilon_{02}=0.07 x_{2}$ relative to the substrate, and also a couple of more complicated variations.

There is a large theoretical and experimental literature on the critical thickness of strained layers, the thickness at which the first misfit dislocations occur (see, e.g., the review by Fitzgerald ${ }^{4}$ ). However, empirically, significant plastic relaxation, or significant change of average in-plane lattice constant, does not occur until a much greater thickness is reached, ${ }^{1,5}$ which we call here the relaxation critical thickness. Between the first critical thickness and the relaxation critical thickness, interfaces may be described as semicoherent. In Ref. 1 we identified a relaxation critical thickness for a layer of misfit $\epsilon_{0}$ of

$$
t_{\mathrm{cr}}=\frac{k}{\epsilon_{0}}
$$

and found that the residual strain at a thickness $d$, greater than relaxation critical thickness is

$$
\epsilon(d)=\frac{k}{d}
$$

until a work-hardened régime is reached at large $d$. The value of $k$ was established experimentally to be $0.8 \pm 0.1 \mathrm{~nm}$ (Ref. 1) and the onset of the work-hardening régime was found to occur at somewhat under $10 t_{\mathrm{cr}}$ (see Fig. 1).

Multilayer structures can be analyzed using Eqs. (1) and (2). We generalize by first introducing the average strain in an $n$-layer structure, given in terms of the individual layer strains $\epsilon_{i}$ and $d_{i}$ as

$$
\dot{\epsilon}_{\mathrm{av}}=\frac{\sum_{i=1}^{n} \epsilon_{i} d_{i}}{\sum_{i=1}^{n} d_{i}}
$$




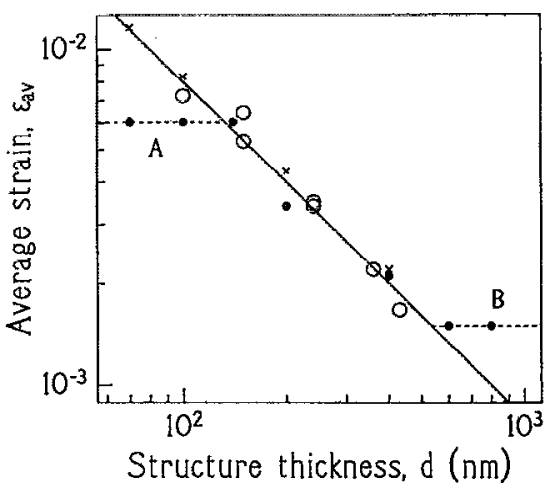

FIG. 1. The residual strain measured by $x$-ray diffraction is plotted against the thickness $d$ for several $\mathrm{In}_{0.1} \mathrm{Ga}_{0.9}$ As samples (solid dots) and $\mathrm{In}_{0.2} \mathrm{Ga}_{0.8}$ As samples (crosses). The $\mathrm{In}_{0.2} \mathrm{Ga}_{0.8}$ As data have been previously published (Ref. 1). The open circles show the average strains given by Eq. (4) in the seven multilayer structures of Table I, plotted against the total thicknesses $d=\Sigma d_{i}$. The solid curve represents $\mathrm{Eq}$. (2) for the single layers and Eq. (4) for the multilayer structures, with $k=0.80 \mathrm{~nm}$. The broken curve at $A$ indicates the misfit strain for the $\operatorname{In}_{0.1} \mathrm{Ga}_{0.9}$ As below critical thickness (plotted for their actual composition of $x=0.09$ ), and the broken curve at $B$ shows the expected work-hardening behavior of these layers.

We now assume that the average strain and the total thickness also follow the relationship of Eq. (2):

$$
\epsilon_{\mathrm{av}}=\frac{k}{\sum_{i=1}^{n} d_{i}}
$$

or, equivalently,

$$
\sum_{i=1}^{n} \epsilon_{i} d_{i}=k .
$$

This model, Eq. (5), can be tested experimentally, and in this letter we present evidence that it is obeyed in relaxing multilayers with the same value of $k, 0.8 \pm 0.1 \mathrm{~nm}$, as in single layers. We may go further, and predict on the basis of both Eqs. (2) and (5) what the strains of the individual layers should be when the structure relaxes. Analysis of the general case of $n$ layers of arbitrary thicknesses and misfit strains is outside the scope of this letter and will be presented elsewhere. The double-layer structures, however, are particularly simple. If the upper layer relaxes relative to the lower layer, it should obey Eq. (2) and thereby use up the entire contribution to $k$ of the strain-thickness products in Eq. (5). It follows at once that the lower layer must be completely relaxes, with zero strain.
This offers an interesting possibility. Independently of the thickness of the first layer, whether it is above or below its own relaxation critical thickness, the strain in it will reach zero when the second layer reaches relaxation critical thickness for the strain corresponding to the difference in misfit strain between the two layers, $\Delta \epsilon=\epsilon_{01}-\epsilon_{02}$,

$$
d_{2}=t_{\mathrm{cr} 2}=\frac{k}{\Delta \epsilon} \text {. }
$$

The physical interpretation is that the second layer can pull the first layer down to zero strain without requiring the infinite thickness otherwise required by Eq. (2), and the concomitant work hardening or degradation of crystalline quality. At this point, the second layer will have the strain $\Delta \epsilon$ and will be at the critical thickness for a layer of this strain. It is ready to relax, breaking the semicoherence of the interface, if it were grown thicker or if a more highly strained layer were to be grown on top.

These theoretical results were tested in some doublelayer structures, and two three-layer structures. Sample growth was done by metallo-organic chemical vapor deposition (MOCVD) and by molecular beam epitaxy (MBE) under various conditions. First, a series of single layers of $\mathrm{In}_{0.1} \mathrm{Ga}_{0.9}$ As were grown under the conditions previously used to demonstrate the single relaxation curve in $\mathrm{In}_{0.2} \mathrm{Ga}_{0.8}$ As single layers ${ }^{1}$ with misfit strain $\epsilon_{0}=0.014$, to check the applicability of Eq. (2) to this alloy composition (misfit strain $\epsilon_{0}=0.007$ ). Double-crystal x-ray diffraction rocking curves for the 004 and 115 reflections along all four 110 directions were used to measure the composition and the strain of the layers (see Ref. 6 for further details). Thicknesses were checked by transmission electron microscopy using cleaved wedge samples. The results are plotted in Fig. 1 together with the $\operatorname{In}_{0.2} \mathrm{Ga}_{0.8}$ As data previously reported; ${ }^{1}$ excellent agreement with $\mathrm{Eq}$. (2) is observed up to the onset of work hardening around $600 \mathrm{~nm}$. The results give a critical thickness for relaxation at this composition of $120 \mathrm{~nm}$.

One double-layer structure (S1 in Table I) was grown by molecular beam epitaxy (MBE) in the same reactor under the same conditions as the single layers, and another (UPM159) was grown under similar conditions in a different MBE reactor, logether with a three-layer structure (UPM162). Another double-layer sample (CNM1) and a three-layer sample (CNM2) were grown by atomic layer MBE (ALMBE) at a much lower growth temperature of $350^{\circ} \mathrm{C}$. Finally, two double-layer samples were grown by MOCVD. Details of

\begin{tabular}{|c|c|c|c|c|c|c|c|c|c|c|}
\hline Sample & $x_{1}$ & $d_{1}(\mathrm{~nm})$ & $\epsilon_{1} \%$ & $x_{2}$ & $d_{2}(\mathrm{~nm})$ & $\epsilon_{2} \%$ & $x_{3}$ & $d_{3}(\mathrm{~nm})$ & $\epsilon_{3} \%$ & $\epsilon_{\mathrm{av}}$ \\
\hline S1 & 0.10 & 120 & $0.25(5)$ & 0.20 & 120 & $0.45(5)$ & & & $0.35(5)$ & \\
\hline CNM1 & 0.10 & 120 & $0.09(1)$ & 0.18 & 120 & $0.58(4)$ & & & & $0.34(4)$ \\
\hline CNM2 & 0.09 & 120 & $0.064(4)$ & 0.18 & 120 & $0.11(1)$ & 0.29 & 120 & $0.49(2)$ & $0.22(2)$ \\
\hline UPM159 & 0.08 & 50 & $0.45(5)$ & 0.29 & 50 & $1.0(5)$ & & & & $0.725(5)$ \\
\hline UPM 162 & 0.085 & 50 & $0.43(5)$ & 0 & 50 & 0.0 & 0.27 & 50 & $1.16(5)$ & $0.53(5)$ \\
\hline EPI239 & 0.14 & 100 & $0.44(5)$ & 0.26 & 50 & $1.05(5)$ & & & & $0.645(5)$ \\
\hline EPI744 & 0.20 & 230 & $0.168(5)$ & 0.34 & 200 & $0.167(5)$ & & & & $0.167(5)$ \\
\hline
\end{tabular}
the structures are given in Table I. The thickness of each

TABLE I. Multilayer structures. 
layer in S1, CNM1, and CNM2 was chosen to test the argument leading to Eq. (6). The other structures provide a test of Eq. (5) over a range of layer thicknesses.

The strains in the individual layers of each structure were measured by double crystal $x$-ray diffraction ${ }^{6}$ and, where necessary, by reciprocal space mapping in a triplecrystal diffractometer. Details of these measurements will be given elsewhere (see also Refs. 7 and 8). The individual layers do not always have the strains predicted by Eq. (6). Some of the data show more strain in the underlying layers and less strain in the top layer than predicted. However, the key result is that the average strain $\epsilon_{\mathrm{av}}$ given by Eq. (3) for all the structures obeys Eq. (5) within error. Each structure as a whole has the average strain predicted by the single relaxation curve (Table I and Fig. 1). When less relaxation has occurred in the lower layers, more relaxation has occurred in the upper layer to compensate. This means, of course, that the interface between the layers cannot remain coherent or semicoherent. Transmission electron microscopy of the first double-layer sample, S1, does indeed show misfit dislocations at this interface as well as at the $\mathrm{GaAs} / \mathrm{In}_{0.1} \mathrm{Ga}_{0.9}$ As interface (details of the microscopy work will be published elsewhere-see also Ref. 7).

The interpretation is that the misfit dislocations are induced by the strain in the whole structure. Some which should lie in the lower interface are instead pinned at the intermediate or higher interface. They therefore relax less strain energy than if they were in the lower interface. More dislocations are therefore induced. To achieve the strains observed in, e.g., structure S1, for each dislocation which is missing from the lower, substrate interface there must be two that are pinned at the intermediate interface. (The factor of 2 follows from the equal layer thicknesses, $d_{1}=d_{2}$ in this sample.) Instead of some constant dislocation density randomly distributed between the two interfaces according to the pinning probabilities, what the single relaxation law requires is that as long as the summed strain-thickness products exceed $k$ further misfit dislocations will be produced until $\Sigma \epsilon_{i} d_{i}=k$ [Eq. (5)]. Even when dislocations are pinned, and the structure fails to reach its thermodynamic equilibrium state, Eq. (5) still applies.

It is not clear whether the pinning is due to the compositional change at the intermediate interface. Indeed, the mechanism whereby a compositional change could provide a local energy minimum for misfit dislocations is not clear. ${ }^{9}$ From the data in Table $\mathrm{I}$, the scatter in values of $\epsilon_{1}$ suggests that the pinning may be related to details of the growth procedures, but further investigation is required. In contrast, the structures obey the empirical single relaxation curve despite a very wide range of growth conditions and this shows clearly that Eqs. (2) and (5) must be regarded as fundamental, not due to, e.g., kinetic constraints (which would be very different at the low ALMBE and high MOCVD growth temperatures).

A physical interpretation of the application to multilayers can be given which is related to the Matthews forcebalance model of critical thickness. ${ }^{10}$ In this model, the linetension of a misfit dislocation is balanced by the force on the threading section at its end where it turns up to the free surface. The force is given by $F=\sigma b d$, where $\sigma$ is the misfit stress and $b$ is the relevant component of the Burger's vector, and it is obviously quite unimportant how $\sigma$ is distributed over $d$-all that will matter is the integral of the stressthickness product.

These results have profound implications for relaxed buffer layers, in which the objective is to achieve substrate quality crystallinity and morphology at the surface at a different lattice constant from the substrate. We have previously remarked that single layers grown to a great thickness are not suitable as buffer layers since they work harden; this corresponds to loss of crystallinity quality. ${ }^{1,6}$ Nor it is satisfactory to leave residual strain in lower layers of a multilayer buffer layer structure as this may relieve during the growth or processing of a device structure on top-that is, residual strain may leave the buffer layer in an unstable state. ${ }^{3}$ The idea behind the two-layer structures reported here was to see if the second layer can pull the first layer to zero strain, leaving it both stable and free of work hardening; if this would work, a third layer could then bring the second layer to zero strain, and so on. However, if dislocations are liable to become pinned at interfaces, leaving layers with residual strain, this sort of simple stepped-composition buffer layer will not work satisfactorily. Instead, it may be necessary to return to the kind of structure proposed in Ref. 11, in which residual compressive strain in a layer is balanced by tensile strain in the next layer to achieve a zero-net-strain structure. In this kind of structure, moreover, no dislocations have been required to cross an interface during plastic relaxation. Alternatively, grading the layers instead of using abrupt interfaces may avoid the creation of the defects or any other phenomenon responsible for the dislocation pinning, thus allowing the material to be pulled down to zero residual strain. ${ }^{12}$

We are grateful to Epitaxial Products Inc. for the supply of the MOCVD structures. This work was supported financially by the Royal Society and the Science and Engineering Research Council (UK), and by the Commission of the European Communities (ESPRIT Basic Research 6854 BLES programme). We thank Dr. R. Beanland for valuable discussions and Dr. G. Gutierrez for assistance with some of the MBE growth.

${ }^{1}$ D. J. Dunstan, P. Kidd, L. K. Howard, and R. A. Dixon, Appl. Phys. Lett. 59, 3390 (1991).

${ }^{2}$ P. Win, Y. Druelle, A. Cappy, Y. Cordier, J. Favre, and C. Bouillet, Appl. Phys. Lett. 61, 922 (1992).

${ }^{3}$ G. F. Burns and C. G. Fonstad, Appl. Phys. Lett. 61, 2199 (1992).

${ }^{4}$ E. A. Fitzgerald, Mater. Sci. Rep. 7, 87 (1991).

${ }^{5}$ A. V. Drigo, A. Aydini, A. Carnera, F. Genova, C. Rigo, C. Ferrari, P. Franzosi, and G. Salviati, J. Appl. Phys. 66, 1975 (1989).

${ }^{6}$ L. K. Howard, P. Kidd, and R. H. Dixon, J. Crys. Growth 125, 281 (1992).

${ }^{7}$ P. Kidd, D. J. Dunstan, R. Grey, J. David, P. F. Fewster, N. L. Andrew, S.I. Molina, and C. J. Kiely, Inst. Phys. Conf. Ser. 134, 321 (1993).

${ }^{8}$ P. F. Fewster and N. L. Andrews, J. Appl. Phys. 74, 3121 (1993).

${ }^{9}$ P. L. Gourley, T. J. Drummond, and B. L. Doyle, Appl. Phys. Lett. 49, 1101 (1986).

${ }^{10}$ J. W. Matthews, S. Mader, and T. B. Light, J. Appl. Phys. 41, 3800 (1970).

${ }^{11}$ D. J. Dunstan, Semicond. Sci. Technol. 6, A76 (1991).

${ }^{12}$ E. A. Fitzgerald, Y.-H. Xie, M. L. Green, D. Brasen, A. R. Kortan, J. Michel, Y.-J. Mii, and B. E. Weir, Appl. Phys. Lett. 59, 811 (1991). 